\title{
The Endogenous Lusitropic and Chronotropic Agent, B-Type Natriuretic Peptide, Limits Cardiac Troponin Release in Cancer Patients with an Early Impairment of Myocardial Relaxation Induced by Anthracyclines
}

\author{
Pierantonio Menna, Emanuela Salvatorelli, Grazia Armento, Ombretta Annibali, Carlo Greco, \\ Francesco Marchesi, Vito Calabrese, Giorgio Reggiardo, and Giorgio Minotti \\ Units of Drug Sciences (P.M., E.S., G.M.), Oncology (G.A.), Hematology (O.A.), Radiation Oncology (C.G.), and Cardiovascular \\ Sciences (V.C.), Department of Medicine and Center for Integrated Research, University Campus Bio-Medico, Rome; \\ Hematology and Stem Cell Transplant Unit, IRCCS Regina Elena National Cancer Institute, Rome (F.M.); and Mediservice S.r.I., \\ Agrate Brianza (Monza), Italy (G.R.)
}

Received August 30, 2018; accepted September 27, 2018

\begin{abstract}
We have reported that cancer patients treated with anthracyclinebased or nonanthracycline chemotherapy developed an early impairment of myocardial relaxation at echocardiography or persistent elevations of the cardiac hormone B-type natriuretic peptide (BNP). Post-hoc pharmacologic analyses showed that BNP elevations were induced by impaired relaxation and caused positive lusitropic effects that maintained normal relaxation. High BNP levels and impaired relaxation were therefore characterized as mutually exclusive manifestations of diastolic dysfunction, but high BNP levels resulted in positive chronotropism and inappropriate tachycardia. Some patients developed increased circulating levels of cardiac troponin I isoform (cTnl), a marker of cardiomyocyte necrosis. Here we have characterized whether cTnl elevations correlated with diastolic dysfunction that manifested as impaired relaxation or a high level of BNP. The effects of high BNP levels on cTnl elevations were also characterized. We show that impaired

relaxation or high BNP levels were significantly more frequent in patients with cTnl elevations. High BNP levels diminished the plasma peak and area under the curve of cTnl, but this result was accompanied by inappropriate tachycardia. cTnl elevations occurred only in patients treated with anthracyclines; moreover, the association of impaired relaxation or high BNP levels with cTnl elevations was significantly more frequent in doxorubicin-treated patients compared with patients treated with its analog, epirubicin. These findings describe cause-and-effect relations between impaired relaxation and cardiomyocyte necrosis, illuminate the role of anthracycline analogs, denote that the beneficial effects of BNP in relieving impaired relaxation and cardiomyocyte necrosis are counterbalanced by inappropriate tachycardia. Patients showing troponin elevations and impaired relaxation or high BNP levels should be treated with lusitropic drugs that lack a positive chronotropism.
\end{abstract}

\section{Introduction}

Cardiotoxicity is a common consequence of cancer therapy (Menna et al., 2008; Zamorano et al., 2016). It may manifest as systolic dysfunction, which is routinely detected by decrements of the left ventricle ejection fraction (LVEF); however, pharmacologic and clinical evidence suggests that systolic dysfunction might be preceded by diastolic dysfunction (Carver et al., 2007; Ewer and Lenihan, 2008; Minotti, 2013; Serrano et al., 2015).

We recently reported that imaging and laboratory indices of cardiotoxicity with a preserved LVEF occurred in otherwise

This study was promoted by Menarini International Operations Luxembourg S.A. and was registered at the European Clinical Trials Database (EUDRACT 2009-016930-29).

https://doi.org/10.1124/jpet.118.253104. asymptomatic patients assessed as early as 1 week after the last cycle of anthracycline- or fluoropyrimidine/platinumbased chemotherapy. Some patients showed impaired myocardial relaxation at echocardiography, whereas others showed persistently increased plasma levels of the cardiac hormone B-type natriuretic peptide (BNP), a marker of ventricular wall stress. Concomitant changes in relaxation and BNP were uncommon (Calabrese et al., 2018). Post hoc pharmacologic analyses allowed us to characterize that 1) impaired relaxation caused cardiomyocytes to release prohormone BNP, which was cleaved into BNP by circulating proteases; and 2) persistent elevations of BNP then mitigated impaired relaxation before it could be detected by echocardiography (Menna et al., 2018). The cardiac relaxant effect of $\mathrm{BNP}$, referred to as positive lusitropic effect, could therefore explain how BNP persistent elevations and impaired relaxation are mutually exclusive manifestations of early diastolic

ABBREVIATIONS: AUC, area under the curve; BNP, B-type natriuretic peptide; cTnl, cardiac troponin isoform I; DT, mean deceleration time of early filling velocity; $E / A$, mean ratio of peak early filling ( $E$ wave) to late diastolic filling (A wave); HR, heart rate; LOQ, limit of quantification; LVEF, left ventricular ejection fraction; Nt-proBNP, aminoterminal fragment of B-type natriuretic peptide prohormone; ULN, upper limit of normal. 
dysfunction. Transient elevations of BNP also occurred during chemotherapy; however, BNP transients were not as effective as BNP persistent elevations in preventing the appearance of impaired relaxation at echocardiography (Menna et al., 2018).

The pharmacologic foundations of BNP lusitropy rest with the activation of receptor-associated guanylyl cyclase, leading to cGMP formation and protein kinase G-dependent modulation of the passive force and stiffness that cardiomyocytes generate in diastole through the giant protein titin (Bishu et al., 2011). In accordance with these notions, defects in the BNP-protein kinase $\mathrm{G}$ signaling cause diastolic dysfunction (van Heerebeek et al., 2012). BNP can also mobilize endothelial C-type natriuretic peptide, which in turn improves myocardial relaxation by diminishing interactions between $\mathrm{Ca}^{2+}$ and myofilaments in diastole (Hillock et al., 2008; Moltzau et al., 2014). Interestingly, however, we showed that BNP persistent elevations increased heart rate (HR) in cancer patients treated by chemotherapy (Menna et al., 2018). Tachycardia was likely due to BNP activation of L-type $\mathrm{Ca}^{2+}$ current in sinoatrial node (Springer et al., 2012). Such inappropriate tachycardia was considered to partly counteract the beneficial effects of BNP in relieving myocardial relaxation (Menna et al., 2018).

In our study of early diastolic dysfunction from cancer drugs, some patients also developed increased plasma levels of troponin, a marker of cardiomyocte necrosis (Calabrese et al., 2018). In oncologic settings, troponin elevations were correlated to a risk of systolic dysfunction and LVEF decrements induced by cancer chemotherapy (Cardinale et al., 2006); however, other studies showed that troponin elevations could correlate equally well with diastolic dysfunction (Kilickap et al., 2005). Correlations between increased troponin levels and diastolic dysfunction were also observed in non-oncologic settings (El Saiedi et al., 2018).

Microvascular dysfunction and loss of $\mathrm{Ca}^{2+}$ homeostasis are important determinants of diastolic dysfunction. Microvascular dysfunction causes subclinical ischemia, which increases $\mathrm{Ca}^{2+}$ levels in cardiomyocytes. Calcium overload then promotes myofilament activation and myocardial stiffness in diastole, which also causes subclinical ischemia, as well as energy dissipation (Stone, 2008; Lovelock et al., 2012; Minotti, 2013; Redfield, 2016). The aforesaid reports of correlations between diastolic dysfunction and troponin elevations suggest that a vicious cycle between microvascular dysfunction, loss of $\mathrm{Ca}^{2+}$ homeostasis, and impaired relaxation may eventually cause some cardiomyocytes to die. These facts offer a rationale for further post hoc analyses that explore interactions between diastolic dysfunction, BNP, and troponin in cancer patients. We characterized whether BNP, being an endogenous lusitropic agent that relieves impaired relaxation, could also diminish cardiac troponin release induced by chemotherapy.

\section{Experimental Procedures}

Patients. All data derived from our pilot prospective, multicenter, real-life study of the incidence of early diastolic dysfunction induced by anthracycline-based chemotherapy (adjuvant treatment of breast cancer, frontline therapy for non-Hodgkin lymphoma) or fluoropyrimidine-platinum chemotherapy (adjuvant treatment of colorectal cancer) (Calabrese et al., 2018). The source population included 80 comorbid-free patients with normal LVEF and diastolic function at baseline. Kidney function was normal for all patients, which was important to avoiding confounding effects attributable to an altered renal clearance of troponin (Fridén et al., 2017). Patients were evaluated at baseline (T0) and 1 week after the last chemotherapy administration (T1). BNP and troponin were measured at T0 and T1. Intertreatment measurements of BNP and troponin were available for a study subgroup of 67 patients. Source population and study subgroup were balanced for demographic, oncologic, and cardiovascular characteristics; both groups showed a prevalence of patients with breast cancer or lymphoma and, hence, a prevalence of anthracycline-based regimens over nonanthracycline regimens (90\% vs. $10 \%$, respectively) (Menna et al., 2018). The study protocol was approved by the ethics committee of each participating clinical center. Written informed consent was obtained from all patients.

BNP and Troponin Assays. BNP was measured by the circulating levels of the aminoterminal fragment of prohormone BNP (Nt-proBNP), which is formed in a (1:1) ratio to BNP. Although hemodynamically inactive compared with BNP, Nt-proBNP shows a remarkably longer half-life (Weber and Hamm, 2006), which helps improve BNP detection and exploit its diagnostic and prognostic value in cardiovascular diseases (Masson et al., 2006; Omland et al., 2007). Troponin was measured by the circulating levels of its cardiac isoform I (cTnI). Intertreatment blood samples were taken at each chemotherapy cycle before infusions were started. This was done for practical reasons; in fact, most patients were treated on an outpatient basis and left the cancer center immediately after infusions ended. Postinfusion samples were available for only two patients who received chemotherapy on an inpatient basis. We also considered that postinfusion levels of BNP would be artifactually increased by fluid overload and myocardial stretch (Menna et al., 2018). All pharmacologic analyses were therefore conducted by considering preinfusion levels of cTnI and BNP.

Both BNP and cTnI were measured in heparin-lithium plasma by Siemens immune-chemiluminescence assay for the Dimension Vista System (Luminescent Oxygen Channeling Immunoassay Technology, Erlangen, Germany). All assays were centralized at the clinical pharmacology unit of University Campus Bio-Medico of Rome. Nt-pro-BNP assay was linear over $5-35.000 \mathrm{pg} / \mathrm{ml}$, and the upper limit of normal (ULN) was set at $125 \mathrm{pg} / \mathrm{ml}$ (Calabrese et al., 2018). Where indicated, a few patients presented at T1 with BNP $>$ ULN that was considered normal owing to noncardiac factors (e.g., fever) (Menna et al., 2018). cTnI assay was linear over $0.015-40 \mathrm{ng} / \mathrm{ml}$. The manufacturer-recommended ULN was rounded from 0.045 to $0.05 \mathrm{ng} / \mathrm{ml}$, and values below the limit of quantification (LOQ) were arbitrarily set at $0.01 \mathrm{ng} / \mathrm{ml}$ (Calabrese et al., 2018).

Other Conditions. Systolic function was measured by LVEF using two-dimensional echocardiography. Values $\geq 50 \%$ denoted a preserved LVEF (Calabrese et al., 2018). Echocardiographic evaluation of diastolic dysfunction was performed by measuring indices of transmitral flow [the mean ratio of peak early filling ( $\mathrm{E}$ wave) to late diastolic filling (A wave), the E/A ratio, and mean deceleration time of early filling velocity (DT)]. All values were normalized to age-related ranges of normality to permit comparisons between patients of different ages. Diastolic dysfunction was adjudicated at T1 by abnormalities of transmitral flow or BNP levels > ULN. All patients with transmitral flow abnormalities showed grade I diastolic dysfunction (impaired relaxation, defined as low-for-age E/A ratio and longfor-age DT) (Nagueh et al., 2009). Higher -grade diastolic dysfunction was not observed (Calabrese et al., 2018).

Other Conditions and Statistical Analyses. Results were expressed as medians with interquartile ranges, or numbers and percentages, and were analyzed by Kruskal-Wallis one-way analysis of variance with Dunn's post-hoc test, two-tailed Mann-Whitney test, or Wilcoxon matched pair test, as appropriate. Categorical variables were analyzed by $\chi^{2}$ test or Fisher's exact test. A value of $P<0.05$ was considered statistically significant. Areas under the curve and best fitting curves were obtained by Prism 5, version 5.01 (GraphPad Software Inc., La Jolla, CA). Fitting curves were accepted when the goodness of fit ( $R^{2}$ coefficient) was $\geq 0.8$ (Menna et al., 2018). For patients exposed to different anthracyclines, (doxorubicin, epirubicin), the cumulative anthracycline dose was expressed as doxorubicin myelotoxic equivalents (Ewer, 2013). Details about 
TABLE 1

Distribution of cardiac troponin isoform I (cTnI) across the LOQ and ULN at T0 and T1 in source population and study subgroup

\begin{tabular}{|c|c|c|c|c|}
\hline Time & $\mathrm{cTnI}$ & $\begin{array}{l}\text { Source Population } \\
\quad(n=80)\end{array}$ & $\begin{array}{c}\text { Study Subgroup } \\
(n=67)\end{array}$ & $P$ (Source Population vs. Study Subgroup) \\
\hline T0 & $\begin{array}{c}<\mathrm{LOQ} \\
>\mathrm{LOQ} / \leq \mathrm{ULN} \\
>\mathrm{ULN}\end{array}$ & $\begin{aligned} 73 & (91 \%) \\
7 & (9 \%) \\
& -\end{aligned}$ & $\begin{aligned} 62 & (93 \%) \\
5 & (7 \%) \\
& -\end{aligned}$ & 0.795 \\
\hline \multirow[t]{2}{*}{$\mathrm{T} 1$} & $\begin{array}{c}<\mathrm{LOQ} \\
>\mathrm{LOQ} / \leq \mathrm{ULN} \\
>\mathrm{ULN}\end{array}$ & $\begin{array}{c}60(75 \%) \\
16(20 \%) \\
4(5 \%)\end{array}$ & $\begin{array}{c}47(70 \%) \\
16(24 \%) \\
4(6 \%)\end{array}$ & 0.731 \\
\hline & & $\begin{array}{c}\mathrm{P}(\mathrm{T} 0 \text { vs. T1) } \\
<0.001\end{array}$ & $\begin{array}{l}\mathrm{P} \text { (T0 vs. T1) } \\
\quad<0.0001\end{array}$ & \\
\hline
\end{tabular}

Data were analyzed by two-tailed $\chi^{2}$ test.

$\mathrm{LOQ}$, limit of quantification $(0.015 \mathrm{ng} / \mathrm{ml})$; ULN, upper limit of normal $(0.05 \mathrm{ng} / \mathrm{ml})$.

anthracycline-based or fluoropyrimidine-platinum regimens are given in the legend to Table 2. Other details are also given in legends for Figures and Tables.

\section{Results}

cTnI Levels at T0 and T1. At T0, $<10 \%$ of patients of source population or study subgroup had cTnI levels $>$ LOQ. At $\mathrm{T} 1$, the percentage of patients with $\mathrm{cTnI}>\mathrm{LOQ}$ increased to $\geq 20 \%$, with few patients showing cTnI $>$ ULN. Source population and study subgroup were balanced for cTnI distribution across LOQ and ULN at T0 and T1; moreover, for each patient group, cTnI distribution across LOQ and ULN at T1 was significantly different compared with T0 (Table 1).

Effects of BNP on cTnI Distribution across LOQ and ULN. At T0, BNP was $<$ ULN in both the source population and study subgroup [pg of Nt-proBNP/ml, medians with interquartile ranges of 60 (37-87) and 60 (37-94), respectively]. At T1, 15 patients of the source population (19\%) and 12 patients of study subgroup (18\%) showed BNP levels $>$ ULN [pg of Nt-proBNP/ml, medians with interquartile ranges of 219 (171-327) and 239 (175-347) $\mathrm{pg} / \mathrm{ml}, P<0.0001$ for T1 vs. T0 in each patient group]. In the source population, BNP elevations occurred in 14 of 72 patients treated by anthracycline-based regimens and in one of eight patients treated by nonanthracycline chemotherapy ( $19 \%$ vs. $13 \%, P=$ $0.335)$. In the study subgroup, BNP elevations occurred in 11 of 60 patients treated by anthracyclines and in one of seven patients treated by nonanthracycline chemotherapy ( $18 \%$ vs. $14 \%, P=0.563$ ). In both source population and study subgroup, the changes in cTnI distribution across LOQ and ULN occurred only if patients presented at $\mathrm{T} 1$ with normal BNP levels. Patients with BNP > ULN showed the same cTnI distribution as that measured at T0 (Fig. 1).

Transient and Persistent Elevations of cTnI. Intertreatment preinfusion measurements of $\mathrm{cTnI}$ were available for the 67 patients of the study subgroup. Among these patients, 1) 51 showed cTnI levels $<$ ULN from T0 to T1; 2) 12 patients showed cTnI levels that exceeded ULN during chemotherapy but declined before T1; and 3) four patients showed cTnI levels that increased during chemotherapy and were $>$ ULN at T1. Thus, 16 of 67 patients showed transient or persistent elevations of cTnI. Only two patients received cTnI assays both before chemotherapy infusion and over the subsequent 24-48 hours. Postinfusion levels of cTnI were not different from preinfusions levels in these patients (Fig. 2). It is worth noting that cTnI began to increase when patients had received $\leq 50 \%$ of their assigned chemotherapy, an interval over which myocardial relaxation began to decline (see also Fig. 2 and insets).

Effects of BNP on Transient or Persistent Elevations of cTnI. Eight of 12 patients with transient cTnI elevations showed intertreatment levels of BNP lower than ULN or only occasionally greater than the ULN (Fig. 3A). Four other patients with transient cTnI elevations showed BNP levels that increased during chemotherapy and exceeded the ULN at $\mathrm{T} 1$. In these latter patients, $\mathrm{cTnI}$ elevations were of a limited magnitude compared with patients with normal BNP at T1 (Fig. 3B).

Three patients with persistent cTnI elevations showed BNP levels below ULN from T0 to T1 (Fig. 4A). One other patient with a persistent cTnI elevation showed BNP levels that increased early during chemotherapy and remained $>$ ULN at
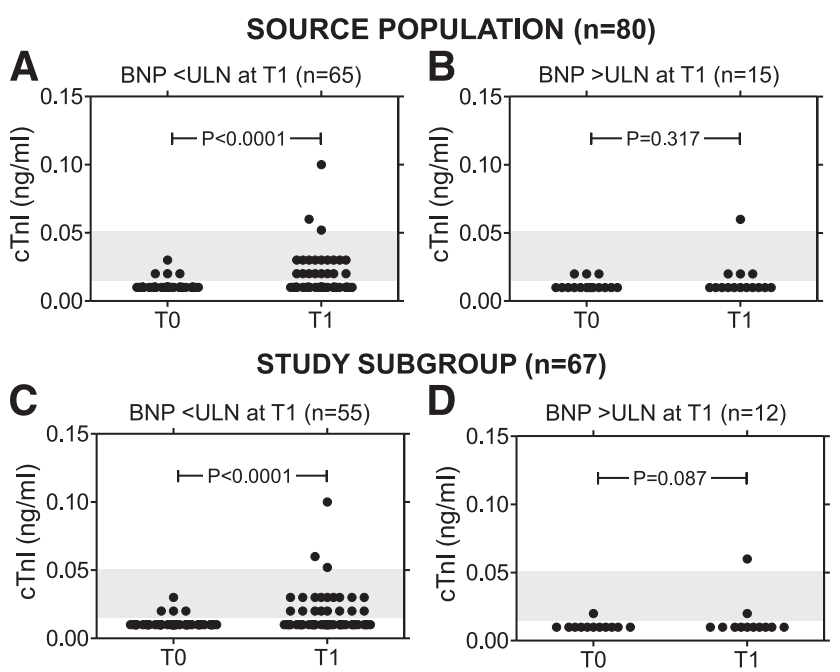

Fig. 1. cTnI distribution across $L O Q$ and ULN in patients with or without BNP $>$ ULN at T1. Patients of source population or study subgroup were stratified according to BNP levels at T1. (A and B) For the source population, chemotherapy caused significant changes in $\mathrm{c} T \mathrm{~T}$ I distribution in patients with BNP $<$ ULN but not in patients with BNP $>$ ULN. The same occurred for patients of the study subgroup (C and D). Shaded areas indicate ranges of cTnI between LOQ $(0.015 \mathrm{ng} / \mathrm{ml})$ and ULN $(0.05 \mathrm{ng} / \mathrm{ml})$. $\mathrm{BNP}$ ULN was set at $125 \mathrm{pg}$ of Nt-proBNP/ml. Data were analyzed by two-tailed $\chi^{2}$ test. 

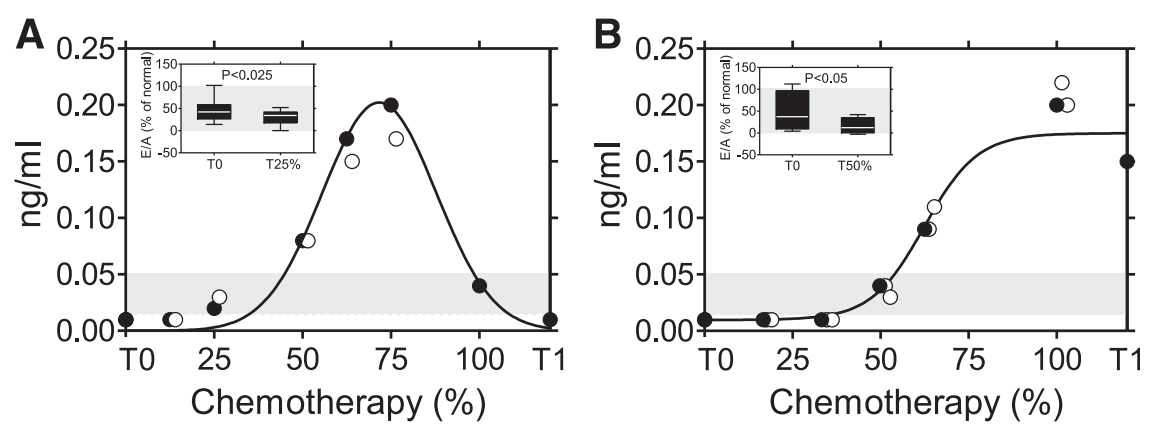

Fig. 2. Preinfusion and postinfusion cTnI levels. (A) Data from a patient with a transient cTnI elevation. (B) Data from a patient with a persistent cTnI elevation. Solid circles denote preinfusion levels of cTnI. Open circles denote cTnI levels measured 24 or 48 hours after infusion. Shaded areas indicate ranges of cTnI between LOQ $(0.015 \mathrm{ng} / \mathrm{ml})$ and ULN $(0.05 \mathrm{ng} / \mathrm{ml})$. Chemotherapy was expressed as the percentage of the length to permit comparisons among oncologic regimens of different durations (eight cycles every 3 weeks in (A), six cycles every 3 weeks in (B)). Insets show the significant decline of myocardial relaxation, measured as decrements of the E/A ratio, that occurred from T0 to T25\% or from T0 to T50\% for 12 patients with transient cTnI elevations (A) or four patients with persistent cTnI elevations (B). Data are shown as Tukey whiskers with medians and interquartile ranges and were analyzed by the Wilcoxon matched paired test. The shaded area indicates the age-adjusted range of E/A normality. Similar results were obtained by expressing impaired relaxation as DT prolongation (data not shown). T25\% or T50\%, median time to completing $25 \%$ or $50 \%$ of the assigned chemotherapy regimen.

T1. This patient showed the least evident deviation of $\mathrm{cTnI}$ from the range of normality (Fig. 4B).

Effects of BNP on Plasma Exposure to cTnI. Data from transient or persistent $\mathrm{cTnI}$ elevations were pooled and used to calculate plasma exposure to $\mathrm{cTnI}\left(C_{\max }\right.$ and area under the curve, AUC). Next, $C_{\max }$ and AUC were correlated to the levels of BNP from T0 to T1 (normal, transiently elevated, persistently elevated). Both $C_{\max }$ and AUC were significantly lower in patients with BNP persistent elevations compared with patients with normal or only transiently high BNP levels (Fig. 5).

Anthracycline Dependence of cTnI Elevations and Diastolic Dysfunction. Patients with or without cTnI elevations were balanced for demographic but not for oncologic
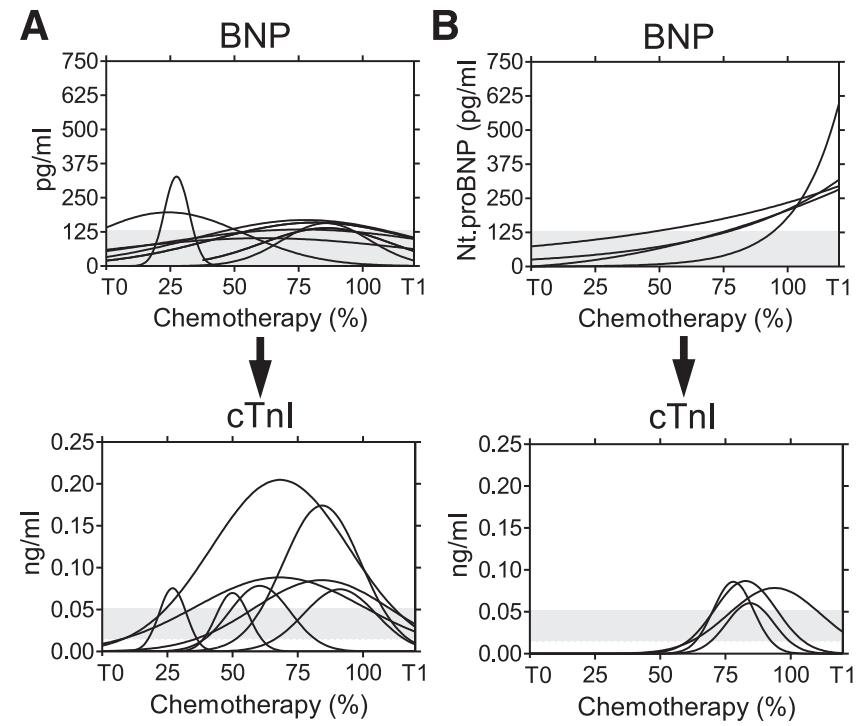

Fig. 3. Time courses of BNP versus transient cTnI elevations. (A) Transient cTnI elevations in patients with intertreatment BNP $<$ ULN or only occasionally $>$ ULN. (B) Transient cTnI elevations in patients who developed gradual and persistent BNP increases. Shaded areas indicate ranges of normality for BNP (ULN at $125 \mathrm{pg}$ of $\mathrm{Nt}-\mathrm{proBNP} / \mathrm{ml}$ ) or ranges between LOQ and ULN for cTnI ( 0.015 and $0.05 \mathrm{ng} / \mathrm{ml}$, respectively). Chemotherapy was expressed as the percentage of the length to permit comparisons among oncologic regimens of different durations. characteristics; in fact, cTnI elevations occurred in patients with breast cancer or non-Hodgkin lymphoma, both requiring anthracycline-based regimens, but not in patients exposed to fluoropyrimidine-platinum regimens for treatment of colorectal cancer. Patients with or without cTnI elevations were nonetheless balanced for cumulative anthracycline dose (Table 2). Forty of 43 patients with breast cancer, accounting for $60 \%$ of all patients of the study subgroup, received also a taxane (docetaxel or paclitaxel, $n=39$ or $n=1$, respectively). Patients with or without cTnI elevations were balanced for taxane administration (see also Table 2 and its legend for details about chemotherapy regimens).

Patients with or without cTnI elevations were balanced for LVEF, systolic blood pressure, diastolic blood pressure, and body mass index. These parameters were normal for both
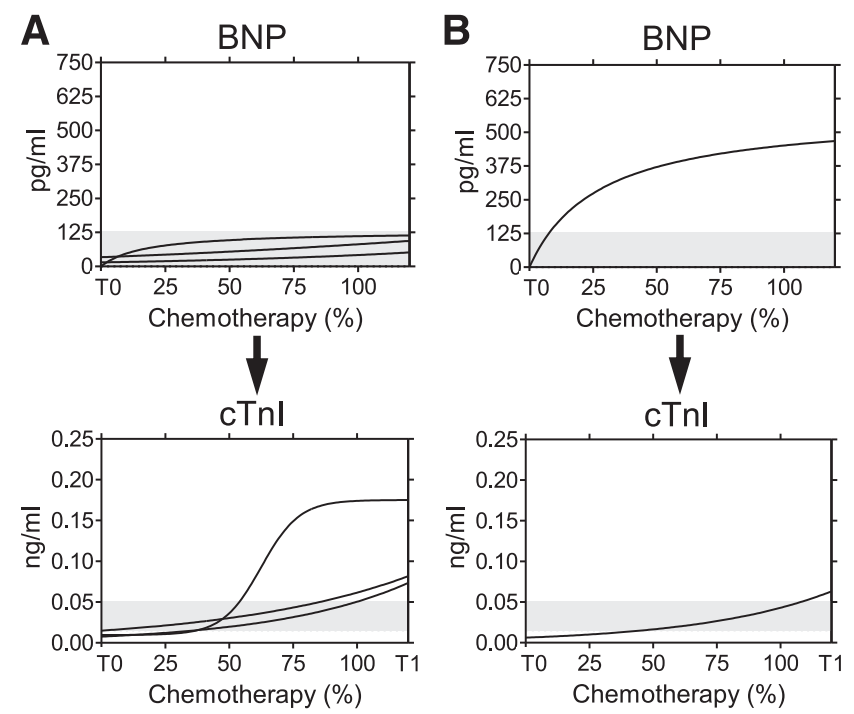

Fig. 4. Time courses of BNP versus persistent cTnI elevations. (A) Persistent cTnI elevations in patients with intertreatment BNP $<$ ULN. (B) Persistent cTnI elevation in a patient who developed BNP levels $>$ ULN. Shaded areas indicate ranges of normality for BNP (ULN at $125 \mathrm{pg}$ of Nt-proBNP/ml) or ranges between LOQ and ULN for $\mathrm{cTnI}(0.015$ and $0.05 \mathrm{ng} / \mathrm{ml}$, respectively). Chemotherapy was expressed as the percentage of the length to permit comparisons among oncologic regimens of different durations. 

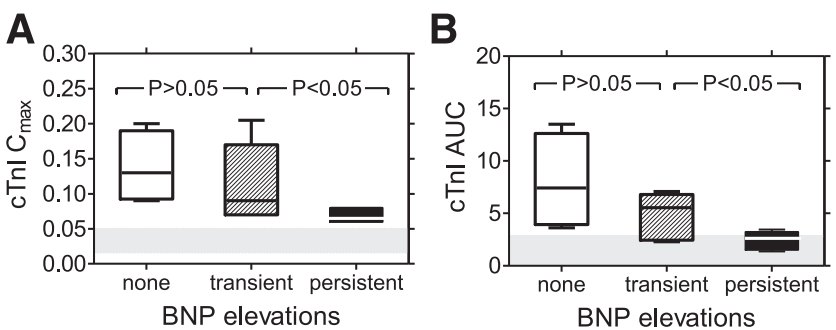

Fig. 5. Plasma exposure to cTnI in patients with different BNP levels from T0 to T1. The $C_{\max }$ and AUC of cTnI were calculated for transient or persistent cTnI elevations in aggregate. BNP elevations were defined as none $(\mathrm{BNP}<\mathrm{ULN}$ from T0 and T1), transient (BNP $>$ ULN during chemotherapy and $<\mathrm{ULN}$ at T1), persistent (BNP $>$ ULN during chemotherapy and at T1). BNP ULN was set at $125 \mathrm{pg}$ of Nt-proBNP/ml. $C_{\max }$ was expressed as nanograms per milliliter, AUC was expressed as nanomoles $\bullet$ (T0-T1). Data are shown as Tukey whiskers with medians and interquartile ranges and were analyzed by Kruskall-Wallis one-way analysis of variance with Dunn's post-hoc test. A) Shaded area indicates the range between LOQ and ULN for cTnI $(0.015$ and $0.05 \mathrm{ng} / \mathrm{ml}$, respectively). (B) Shaded area indicates the range of normality for $\mathrm{cTnI}$ AUC (calculated for patients with BNP $<$ ULN from T0 to T1).

groups at $\mathrm{T} 0$ and did not change at $\mathrm{T} 1$; interestingly, however, patients with cTnI elevations were diagnosed at T1 with a higher incidence of diastolic dysfunction (impaired relaxation or persistently high BNP) (Table 3). The group of patients with a lower incidence of diastolic dysfunction and no $c \mathrm{TnI}$ elevation included 44 subjects exposed to anthracyclines and seven subjects exposed to nonanthracycline chemotherapy. We mentioned that one case of BNP elevation occurred in the nonanthracycline group, but another patient in the same group developed an impaired relaxation. When the nonanthracycline group was excluded from analyses, anthracycline-treated/cTnIpositive patients still exhibited a higher incidence of diastolic dysfunction compared with anthracycline-treated/cTnI negative patients $(9 / 16$ vs. $15 / 44, P<0.01)$.

For patients treated with anthracyclines and showing cTnI elevations, diastolic dysfunction could equally well manifest as impaired relaxation $(n=4 / 9)$ or $\mathrm{BNP}>\mathrm{ULN}(n=5 / 9)$.
Compared with patients with impaired relaxation, those with BNP > ULN showed normal-for-age E/A and DT and lower $C_{\max }$ and AUC values of cTnI (Fig. 6).

Patients treated with anthracycline-based chemotherapy received doxorubicin $(n=35)$ or its closely related analog, epirubicin $(n=25)$. Neither doxorubicin nor epirubicin caused significant changes in LVEF from $\mathrm{T} 0$ to $\mathrm{T} 1$; moreover, patients treated with doxorubicin or epirubicin were balanced for any cTnI elevation that occurred from T0 to $\mathrm{T} 1$ and for any diastolic dysfunction that was adjudicated at $\mathrm{T} 1$ as impaired relaxation or persistently high BNP. Interestingly, however, a pattern characterized by diastolic dysfunction with concomitant cTnI elevations was significantly more frequent in patients treated with doxorubicin (Table 4).

BNP, cTnI, and Tachycardia. All patients diagnosed at T1 had tachycardia, which was at least in part induced by chemotherapy-related bone marrow toxicity and anemia. Hemoglobin loss and tachycardia were of similar extent for patients with or without cTnI elevations (Table 5). We characterized whether the previously reported chronotropic effect of BNP could be evidenced by stratifying patients for both cTnI and BNP levels. For patients with BNP $<$ ULN at T1, tachycardia (expressed as $\Delta \mathrm{bpm}$ from $\mathrm{T} 0$ to $\mathrm{T} 1$ ) was not influenced by $\mathrm{cTnI}$ elevations (Fig. 7A). For patients with BNP $>$ ULN at T1, higher BNP levels and $\Delta \mathrm{bpm}$ occurred if $\mathrm{cTnI}$ elevations had also occurred (Fig. 7B).

\section{Discussion}

Cause-and-Effect Relations Between Impaired Relaxation and cTnI Elevations. Several lines of evidence point to cause-and-effect relations between diastolic dysfunction and cTnI elevations. Diastolic dysfunction, defined as impaired relaxation or BNP persistent elevations that mitigated impaired relaxation, was more frequent in patients with cTnI elevations (see Table 3). Both a limited distribution of cTnI across LOQ and ULN at T1, as well as lower $C_{\max }$ and

TABLE 2

Demographic and oncologic characteristics of patients with or without transient or persistent cardiac troponin isoform I (cTnI) elevations

\begin{tabular}{|c|c|c|c|}
\hline Characteristics & cTnI- $(n=51)$ & cTnI $+(n=16)$ & $P($ cTnI- vs. cTnI +$)$ \\
\hline Age (yr) & $49(41-56)$ & $52(47-58)$ & 0.143 \\
\hline $\begin{array}{l}\text { Gender }(n, \%) \\
\text { male } \\
\text { female }\end{array}$ & $\begin{array}{r}9(18 \%) \\
42(82 \%)\end{array}$ & $\begin{array}{r}2(13 \%) \\
14(87 \%)\end{array}$ & 0.435 \\
\hline $\begin{array}{l}\text { Oncologic disease }(n, \%) \text { : } \\
\text { breast cancer } \\
\text { non-Hodgkin lymphoma } \\
\text { colorectal cancer }\end{array}$ & $\begin{array}{r}32(63 \%) \\
12(23 \%) \\
7(14 \%)\end{array}$ & $\begin{array}{c}11(69 \%) \\
5(31 \%) \\
0\end{array}$ & 0.001 \\
\hline $\begin{array}{l}\text { Chemotherapy }(n, \%) \text { : } \\
\text { anthracycline-based, } \\
\text { nonanthracycline }^{b}\end{array}$ & $\begin{array}{r}44(86 \%) \\
7(14 \%)\end{array}$ & $\begin{array}{c}16(100 \%) \\
0\end{array}$ & $<0.0001$ \\
\hline Anthracycline dose $\left(\mathrm{mg} / \mathrm{m}^{2}\right)^{c}$ & $240(240-300)$ & $240(240-300)$ & 0.465 \\
\hline Taxane administration $(n, \%)$ & $29(57 \%)$ & $11(69 \%)$ & 0.185 \\
\hline
\end{tabular}

cTnI- or cTnI+ denotes the absence or presence of cTnI elevations.

Values are medians with interquartile ranges. Differences were analyzed by two-tailed Mann-Whitney test, $\chi^{2}$ test, or Fisher's exact test as appropriate.

${ }^{a}$ For breast cancer: doxorubicin (or epirubicin)/cyclophosphamide once every 3 weeks (four cycles), followed by taxane once every 3 weeks (four cycles);

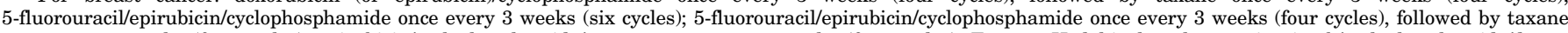

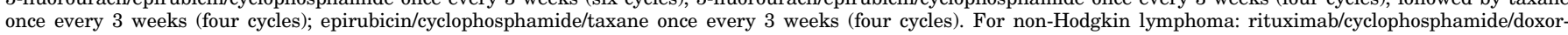
ubicin/vincristine/prednisone once every 3 weeks (six cycles).

${ }^{b}$ Folinate/5-fluouracil/oxaliplatin once every 2 weeks (12 cycles); capecitabine (orally)/oxaliplatin once every 3 weeks (eight cycles).

${ }^{c}$ Doxorubicin myelotoxic equivalents. 
TABLE 3

Cardiovascular characteristics of patients with or without transient or persistent cardiac troponin isoform I (cTnI) elevations

\begin{tabular}{|c|c|c|c|}
\hline Parameter & cTnI- $(n=51)$ & $\mathrm{cTnI}+(n=16)$ & $P($ cTnI- vs. cTnI +$)$ \\
\hline $\begin{array}{l}\text { LVEF (\%): } \\
\text { T0 } \\
\text { T1 } \\
P(\mathrm{~T} 0 \text { - T1) }\end{array}$ & $\begin{array}{c}61(60-65) \\
61(60-65) \\
0.893\end{array}$ & $\begin{array}{c}60(60-65) \\
60(60-65) \\
0.722\end{array}$ & $\begin{array}{l}0.608 \\
0.445\end{array}$ \\
\hline $\begin{array}{l}\text { SBP (mm Hg): } \\
\text { T0 } \\
\text { T1 } \\
P(\mathrm{~T} 0 \text { - T1) }\end{array}$ & $\begin{array}{c}120(110-130) \\
120(110-130) \\
0.957\end{array}$ & $\begin{array}{c}120(120-124) \\
120(115-128) \\
0.928\end{array}$ & $\begin{array}{l}0.818 \\
0.855\end{array}$ \\
\hline $\begin{array}{l}\mathrm{DBP}(\mathrm{mm} \mathrm{Hg}) \\
\text { T0 } \\
\text { T1 } \\
P(\mathrm{~T} 0-\mathrm{T} 1)\end{array}$ & $\begin{array}{c}80(70-80) \\
80(70-80) \\
0.498\end{array}$ & $\begin{array}{c}80(70-80) \\
80(70-85) \\
0.471\end{array}$ & $\begin{array}{l}0.974 \\
0.514\end{array}$ \\
\hline $\begin{array}{l}\mathrm{BMI}\left(\mathrm{kg} / \mathrm{m}^{2}\right): \\
\text { T0 } \\
\mathrm{T} 1 \\
P(\mathrm{~T} 0 \text { - T1) }\end{array}$ & $\begin{array}{l}24(22-25) \\
25(22-28) \\
0.122\end{array}$ & $\begin{array}{c}23(21-25) \\
24(20-26) \\
0.608\end{array}$ & $\begin{array}{l}0.157 \\
0.108\end{array}$ \\
\hline Diastolic dysfunction $^{a}$ & $17(33 \%)$ & $9(56 \%)$ & $<0.001$ \\
\hline
\end{tabular}

BMI, body mass index; DBP, diastolic blood pressure; LVEF, left ventricular ejection fraction; SBP, systolic blood pressure.

cTnI- or cTnI+ denotes the absence or presence of cTnI elevations.

Values are medians with interquartile ranges or absolute numbers with percentages. Differences were analyzed by two-tailed Mann-Whitney test, Wilcoxon matched pair test, or Fisher's exact test as appropriate.

${ }^{a}$ Defined as impaired relaxation at echocardiography or BNP persistent elevations.

AUC values from $\mathrm{T} 0$ to $\mathrm{T} 1$, were documented for patients showing persistent elevations of BNP (see Fig. 1; Figs. 3-6). Finally, transient elevations of BNP did not diminish plasma exposure to cTnI (see Fig. 5), similarly to that reported for the effects of BNP on impaired relaxation (Menna et al., 2018).

Cause-and-effect relations between impaired relaxation and troponin elevations should be weighed against contrasting evidence from the literature. Troponin elevations were in fact attributed to cardiomyocyte necrosis that cancer drugs induced by their own mechanisms, independent of diastolic dysfunction. Troponin levels increased shortly after chemotherapy infusions and declined in few days, which was considered to denote the acute toxicity of cancer drugs to cardiomyocytes (Sandri et al., 2003). Here cTnI elevations were detected before each chemotherapy infusion; hence, they denoted cardiomyocyte necrosis that developed over the weeks that separated consecutive infusions. Moreover, in patients presenting at a chemotherapy cycle with an ongoing elevation of cTnI, administration of chemotherapy did not cause further increases of cTnI (see Fig. 2). Although this information was available for only two patients, this finding denotes that the acute toxicity of chemotherapy infusions did not contribute to the cTnI elevations but reflected an ongoing impairment of myocardial relaxation. Accordingly, both cTnI elevations and a decline of myocardial relaxation occurred early during chemotherapy (see Fig. 2 and insets).

On a different note, troponin elevations were reported to identify patients at risk for LVEF decrements (Cardinale et al., 2006). Here cTnI elevations were not accompanied by LVEF decrements at T1 (see Tables 3 and 4). Having acknowledged that LVEF may decrease months or years after ending chemotherapy (Minotti et al., 2010; Cardinale et al., 2018), we suggest that our findings describe a scenario wherein diastolic dysfunction precedes systolic dysfunction and causes cardiomyocyte necrosis as well.

cTnI, BNP, and Inappropriate Tachycardia. Bone marrow toxicity and anemia are common consequences of chemotherapy. A certain degree of tachycardia is therefore normal for cancer patients treated by chemotherapy (see Table 5). We previously reported that persistent elevations of BNP caused tachycardia independent of anemia (Menna

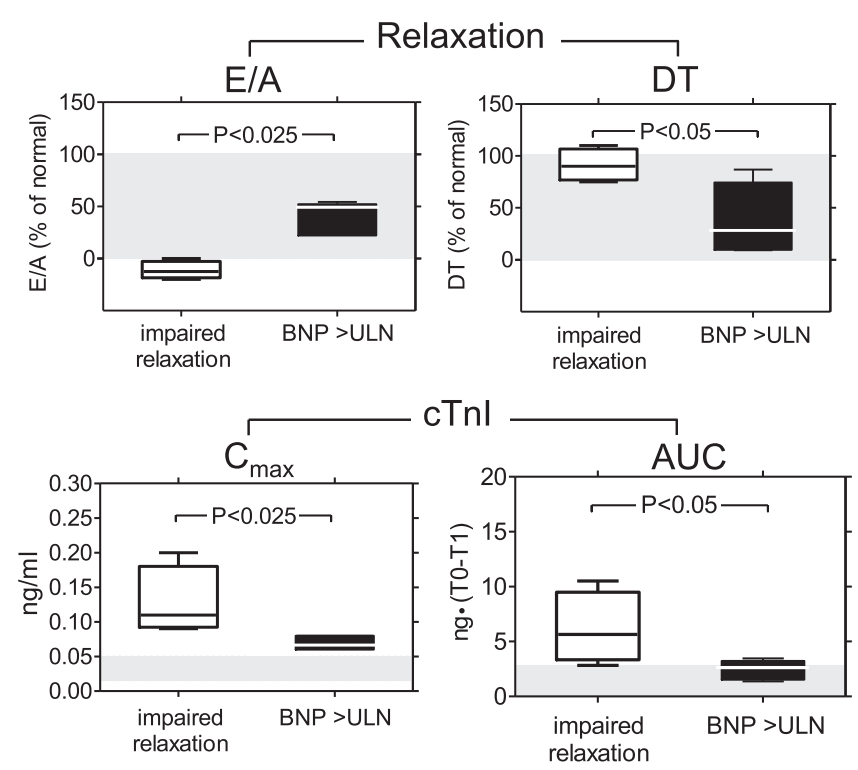

Fig. 6. Plasma exposure to $\mathrm{c} T n I$ in patients with transient or persistent cTnI elevations and diastolic dysfunction at T1. Diastolic dysfunction was adjudicated as impaired relaxation $(n=4)$ or $\mathrm{BNP}>\mathrm{ULN}(n=5)$. BNP ULN was set at $125 \mathrm{pg}$ of Nt-proBNP/ml. The $C_{\max }$ and AUC of cTnI were calculated for transient or persistent $\mathrm{cTnI}$ in aggregate. $C_{\max }$ was expressed as nanograms per milliliter, AUC was expressed as nanomoles`(T0-T1). Data are shown as Tukey whiskers with medians and interquartile ranges and were analyzed using two-tailed Mann-Whitney test. In upper panels, shaded areas denote age-adjusted ranges of normality for the E/A ratio or DT. In the bottom left panel, shaded area denotes ranges between LOQ and ULN for cTnI $(0.015$ and $0.05 \mathrm{ng} / \mathrm{ml}$, respectively). In the bottom right panel, shaded area denotes the range of normality for cTnI AUC between (calculated for patients with BNP $<$ ULN from T0 to T1). 
TABLE 4

Diastolic dysfunction and cardiac troponin isoform I (cTnI) elevations in patients treated with doxorubicin- or epirubicin-based chemotherapy

\begin{tabular}{|c|c|c|c|c|}
\hline Anthracycline & $\Delta \operatorname{LEVF}(\%)^{a}$ & Diastolic Dysfunction (all) $^{b}$ & $\mathrm{cTnI}^{\top}$ Elevations (all) ${ }^{c}$ & Diastolic Dysfunction with cTnI Elevation \\
\hline $\begin{array}{l}\text { Doxorubicin } \\
(n=35)\end{array}$ & $0(-2$ to +2$)$ & $\begin{array}{c}14 \\
(40 \%)\end{array}$ & $\begin{array}{c}9 \\
(26 \%)\end{array}$ & $\begin{array}{c}7 \\
(20 \%)\end{array}$ \\
\hline \multirow[t]{2}{*}{$\begin{array}{l}\text { Epirubicin } \\
(n=25)\end{array}$} & $0(-0.5$ to 1$)$ & $\begin{array}{c}10 \\
(40 \%)\end{array}$ & $\begin{array}{c}7 \\
(28 \%)\end{array}$ & $\begin{array}{c}2 \\
(8 \%)\end{array}$ \\
\hline & $P=0.739$ & $P=1.000$ & $P=0.874$ & $P<0.025$ \\
\hline
\end{tabular}

Data were analyzed by two-tailed Fisher's exact test.

${ }^{a}$ Calculated as [(LVEF T1) - (LVEF T0)].

${ }^{b}$ Impaired relaxation or B-type natriuretic peptide persistent elevations in aggregate.

${ }^{c}$ Any transient or persistent cTnI elevation.

et al., 2018). Here we characterized that such inappropriate tachycardia occurred in those patients in whom high BNP was involved in mitigating both impaired relaxation and cTnI elevations (see Fig. 6 and 7). Having suggested that BNP and cTnI elevations shared impaired relaxation as a causative agent, we also suggest that concomitant elevations of the two biomarkers denoted a condition of increased diastolic distress. Higher BNP levels were therefore required to relieve such distress, causing positive chronotropism and inappropriate tachycardia to occur.

Study Limitations and Pharmacologic Considerations. cTnI elevations occurred in 16 of 60 patients treated with anthracycline-based regimens, but they did not occur in any of seven patients treated with fluoropyrimidine-platinum compounds (see Table 2). Two of seven such patients at T1 showed impaired relaxation at echocardiography or BNP persistent elevations, clearly denoting that nonanthracycline chemotherapeutics were able to induce the two manifestations of diastolic dysfunction. Taking the limited sample size into account, one might argue that cTnI elevations were not caused by impaired relaxation but rather reflected acute toxicity that only anthracyclines could inflict through, for example, oxidative stress (Vejpongsa and Yeh, 2014).

The aforesaid concerns must be weighed against pathophysiologic and pharmacologic considerations. As mentioned, microvascular dysfunction and inappropriate interactions between $\mathrm{Ca}^{2+}$ and myofilaments in diastole are important determinants of impaired myocardial relaxation. Anthracyclines impair endothelial function in cancer patients (Finkelman et al., 2017) and can also reduce the expression and activity of the $\mathrm{Ca}^{2+}$ ATPase that sequesters $\mathrm{Ca}^{2+}$ in sarcoplasmic reticulum during diastole (Minotti et al., 2004). Nonanthracycline chemotherapeutics cause microvascular dysfunction but lack anthracycline-like effects on reducing $\mathrm{Ca}^{2+}$ sequestration (Menna et al., 2008), which may explain how both anthracyclines and nonanthracycline drugs diminished myocardial relaxation at echocardiography or increased BNP levels, whereas only anthracyclines caused a continued diastolic tension that exposed some cardiomyocytes to subclinical ischemia, energy dissipation, and eventually to necrosis. These concepts would be consistent with the so-called type 1-type 2 classification of cancer drugs, in which anthracyclines are type 1 agents that inflict an irreversible damage to cardiomyocytes (Suter and Ewer, 2013).

Pharmacologic insight is also offered by comparing doxorubicin with epirubicin. These anthracyclines were administered in equimyelotoxic doses; yet, doxorubicin was more effective than epirubicin in increasing the number of patients with diastolic dysfunction and concomitant cTnI elevations (see Table 4). This finding was consistent with doxorubicin showing higher levels of accumulation in human myocardium and higher levels of conversion to a secondary alcohol metabolite that mediates inhibition of sarcoplasmic $\mathrm{Ca}^{2+}$ ATPase (Olson et al., 1988; Salvatorelli et al., 2018). Doxorubicin, but not epirubicin, is also susceptible to metabolic interactions with taxanes that were frequently included in anthracycline-based treatment of breast cancer; in fact, taxanes cause allosteric effects that stimulate alcohol metabolite formation from doxorubicin but not epirubicin (Salvatorelli et al., 2007). Thus, doxorubicin is more potent than epirubicin in dysregulating $\mathrm{Ca}^{2+}$ homeostasis, which would expose cardiomyocytes to more sustained and inappropriate interactions between $\mathrm{Ca}^{2+}$ and myofilaments in diastole. These findings also denote that type 1 agents, like anthracyclines, may show important analog-related differences.

TABLE 5

Hemoglobin $(\mathrm{Hb})$ loss and tachycardia in patients with or without transient or persistent cardiac troponin isoform I (cTnI) elevations

\begin{tabular}{lccc}
\hline \multicolumn{1}{c}{ Parameter } & $\mathrm{cTnI}-(n=51)$ & $\mathrm{cTnI}+(n=16)$ & $P(\mathrm{cTnI}-\mathrm{vs.}$ cTnI+ \\
\hline $\mathrm{Hb}(\mathrm{g} / \mathrm{dl}):$ & & & \\
T0 & $13.0(11.8-13.9)$ & $13.3(12.4-14.7)$ & 0.255 \\
T1 & $11.6(10.5-12.3)$ & 0.001 & 0.080 \\
$P(\mathrm{~T} 0-\mathrm{T} 1)$ & $<0.0001$ & & \\
Heart rate $(\mathrm{bpm}):$ & & $76(69-82)$ & 0.994 \\
T0 & $76(70-81)$ & $87(79-100)$ & 0.168 \\
T1 & $85(75-89)$ & $<0.01$ & \\
P $\mathrm{T} 0-\mathrm{T} 1)$ & $<0.01$ & & \\
\hline
\end{tabular}

cTnI- or cTnI+ denotes the absence or presence of cTnI elevations. Data were analyzed by two-tailed Mann-Whitney test or Wilcoxon matched-paired $t$ test as appropriate. $\mathrm{Hb}$, hemoglobin. 

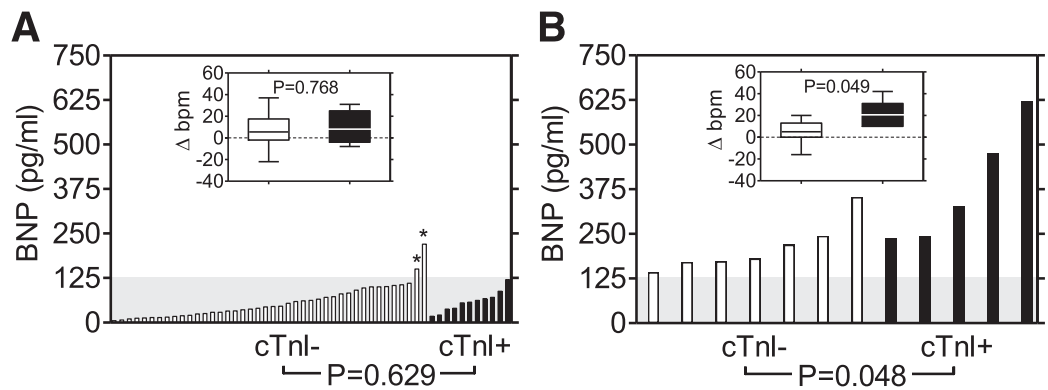

Fig. 7. BNP chronotropisms and cTnI elevations. cTnI- and cTnI+ denote the absence or presence of transient or persistent cTnI elevations in aggregate. Tachycardia was expressed as $\Delta$ bpm from T0 to T1 (medians with interquartile ranges). (A) For patients with BNP $<$ ULN at T1, tachycardia was similar for patients with or without cTnI elevations ( $n=11$ and 44, respectively). (B) For patients with BNP $>$ ULN at T1, both BNP elevations and tachycardia were significantly higher in patients with cTnI elevations $(n=5)$ compared with patients without cTnI elevations $(n=7)(327 \mathrm{pg} \mathrm{vs} .180 \mathrm{pg}$ of BNP/ml, 20 vs. $5 \Delta \mathrm{bpm}$ ). Shaded areas denote the range of normality of BNP (ULN at $125 \mathrm{pg}$ of Nt-proBNP/ml). Asterisks denote patients with BNP $>$ ULN, considered normal by investigators due to noncardiac factors, for example, fever. Data were analyzed by two-tailed Mann-Whitney test.

Clinical Implications. We have shown that anthracyclines cause impaired myocardial relaxation that is accompanied by cTnI release. By relieving impaired relaxation BNP diminishes cTnI release, but this requires BNP levels that eventually induce positive chronotropism and inappropriate tachycardia (see Fig. 8). This scenario anticipates many clinical implications.

Diastolic dysfunction and cTnI elevations occurred in lowrisk patients treated by a median cumulative dose of $240 \mathrm{mg}$ of doxorubicin equivalents $/ \mathrm{m}^{2}$, which was significantly lower than doses associated with $5 \%$ risk of heart failure from doxorubicin or epirubicin $\left(\sim 380-400 \mathrm{mg} / \mathrm{m}^{2}\right.$ or $\sim 600 \mathrm{mg}$ of doxorubicin equivalents $/ \mathrm{m}^{2}$, respectively) (Swain et al., 2003; Salvatorelli et al., 2018). cTnI elevations began to occur early during the chemotherapy regimen (see Figs. 2-4) and were accompanied by a decline in myocardial relaxation. For patients treated with anthracycline-taxane regimens for breast cancer or by the R-CHOP regimen for non-Hodgkin lymphoma, elevations occurred after cumulative doses of $120-150 \mathrm{mg}$ of doxorubicin equivalents $/ \mathrm{m}^{2}$. Although epirubicin merits consideration as a less cardiotoxic analog, these findings show that cardiotoxicity develops after reportedly safe doses of any anthracycline (Salvatorelli et al., 2018).

On a different note, we acknowledge that impaired relaxation represents the mildest degree of diastolic dysfunction, which may or not be accompanied by an increased ventricular filling pressure (Nagueh et al., 2016). We caution that impaired relaxation, if untreated, may progress toward more advanced stages of diastolic dysfunction and eventually toward systolic dysfunction, especially if the following pharmacologic factors are taken into consideration: 1) doxorubicin or epirubicin secondary alcohol metabolites show a limited elimination from cardiomyocytes (Salvatorelli et al., 2018), and 2) cancer survivors may require sequential treatments whose toxicity overlaps with long-lived anthracycline metabolites and eventually precipitates cardiac events (Menna et al., 2008).

Troponin elevations were used to identify patients with early cardiotoxicity and to start therapy with for example, angiotensin-converting enzyme inhibitors; however, these drugs prevented LVEF decrements but not troponin elevations (Cardinale et al., 2018). Here diastolic dysfunction occurred in patients with a preserved LVEF; cause-andeffect relations between impaired relaxation and cTnI elevations were characterized; and persistently high BNP relieved myocardial relaxation and diminished cTnI release. These findings suggest that lusitropic drugs might be considered to treat early cardiotoxicity with cTnI elevations; however, these drugs should lack BNP-like effects on increasing HR.

The antianginal drug ranolazine improves myocardial relaxation but lacks direct effects on HR (Stone, 2008; Lovelock
A

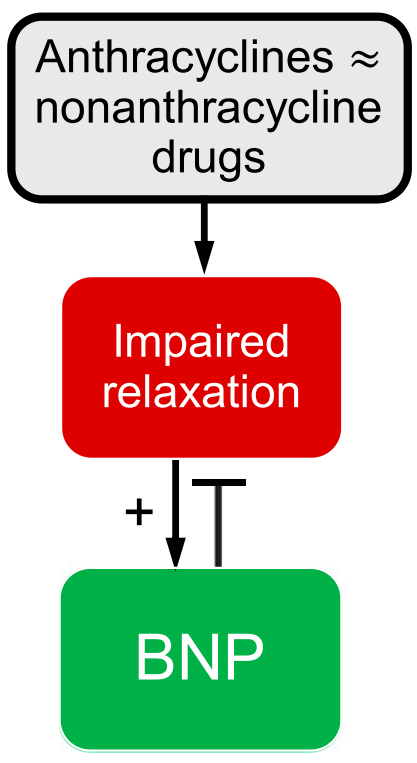

B

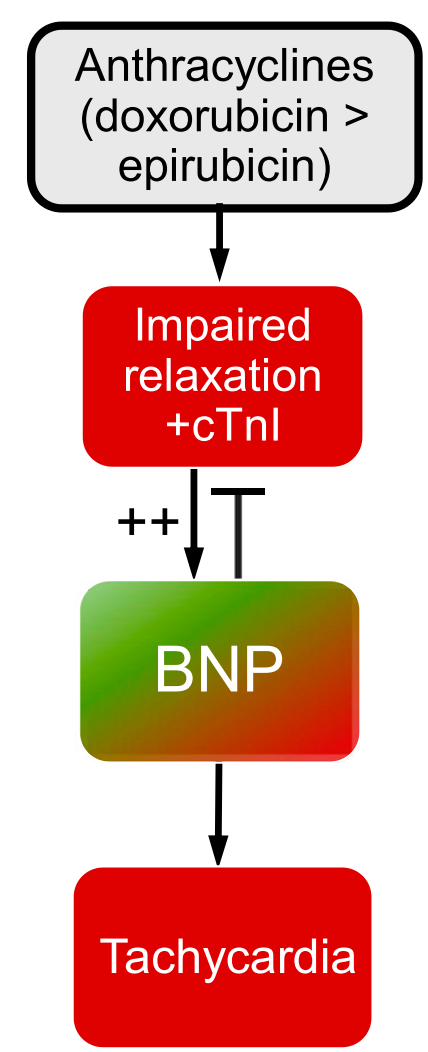

Fig. 8. Schematic representation of multiple interactions between cancer drugs, impaired relaxation, BNP, and cTnI. (A) Both anthracyclines and nonanthracycline chemotherapeutics can cause impaired relaxation, which induces BNP elevations that in turn mitigate impaired relaxation. (B) Only anthracyclines can cause impaired relaxation and concomitant cTnI elevations; doxorubicin is more toxic than epirubicin. This represents a stronger stimulus to BNP elevations, resulting in both beneficial effects (mitigation of impaired relaxation and troponin release) and detrimental side effects (positive chronotropism and inappropriate tachycardia). 
et al., 2012). We reported that ranolazine, being a lusitropic agent, could substitute for BNP in relieving impaired relaxation and, by doing so, diminished BNP levels that otherwise induced inappropriate tachycardia (Menna et al., 2018). Ranolazine effects on cardiac troponin release will be described in the final report of a phase $2 \mathrm{~B}$ study in which patients with chemotherapy-related diastolic dysfunction (defined as impaired relaxation or high BNP or high troponin at T1) were randomized to ranolazine or investigator's choice of angiotensin-converting enzyme inhibitors, $\beta$-blockers, or other common cardiovascular drugs (Minotti, 2013).

Conclusions. BNP has long been used as a biomarker of cardiovascular disease (Braunwald, 2008). Previously, we reported that persistent $\mathrm{BNP}$ elevations relieved impaired myocardial relaxation in patients treated by anthracyclinebased or nonanthracycline chemotherapy. Here we have shown that persistent BNP elevations diminished the circulating levels of cTnI in patients treated with anthracyclines. These findings broaden our perception of BNP as an endogenous modulator of cardiotoxicity.

\section{Acknowledgments}

We thank the participants in the pilot study from which data were originated: Giuseppe Avvisati, Daniele Santini, and Giuseppe Tonini (Campus Bio-Medico University, Rome); Claudio Brunelli and Paolo Spallarossa (San Martino Hospital, Genova); Manuela Rizzo and Maria Cantonetti (Tor Vergata University, Rome); Armando Carpino, Alessandra Fabi, and Giuseppe Toglia (IRCCS Regina Elena National Cancer Institute, Rome); Alessandra D'Ambrosi and Francesco Fedele (La Sapienza University, Rome); Roberto Fiaschetti and Maria Rita Noviello (Grassi Hospital, Ostia, Rome); Laura Massa (University Hospital, Trieste); Nicola Maurea (National Cancer Institute, Naples); and Stefano Oliva (Giovanni XXXIII Cancer Institute, Bari).

\section{Authorship Contributions}

Participated in research design: Menna, Minotti.

Conducted experiments: Menna, Calabrese, Armento, Annibali, Greco, Marchesi.

Contributed new reagents or analytic tools: Menna.

Performed data analysis: Salvatorelli, Reggiardo, Minotti.

Wrote or contributed to the writing of the manuscript: Minotti.

\section{References}

Bishu K, Hamdani N, Mohammed SF, Kruger M, Ohtani T, Ogut O, Brozovich FV, Burnett JC Jr, Linke WA, and Redfield MM (2011) Sildenafil and B-type natriuretic peptide acutely phosphorylate titin and improve diastolic distensibility in vivo. Circulation 124:2882-2891.

Braunwald E (2008) Biomarkers in heart failure. N Engl J Med 358:2148-2159.

Calabrese V, Menna P, Annibali O, Armento G, Carpino A, Cerchiara E, Greco C, Marchesi F, Spallarossa P, Toglia G, et al.; Collaborators (2018) Early diastolic dysfunction after cancer chemotherapy: primary endpoint results of a multicenter cardio-oncology study. Chemotherapy 63:55-63.

Cardinale D, Ciceri F, Latini R, Franzosi MG, Sandri MT, Civelli M, Cucchi G, Menatti E, Mangiavacchi M, Cavina R, et al.; ICOS-ONE Study Investigator (2018) Anthracycline-induced cardiotoxicity: a multicenter randomised trial comparing two strategies for guiding prevention with enalapril: the International CardioOncology Society-one trial. Eur J Cancer 94:126-137.

Cardinale D, Colombo A, Sandri MT, Lamantia G, Colombo N, Civelli M, Martinelli G, Veglia F, Fiorentini C, and Cipolla CM (2006) Prevention of high-dose chemotherapyinduced cardiotoxicity in high-risk patients by angiotensin-converting enzyme inhibition. Circulation 114:2474-2481.

Carver JR, Shapiro CL, Ng A, Jacobs L, Schwartz C, Virgo KS, Hagerty KL Somerfield MR, and Vaughn DJ; ASCO Cancer Survivorship Expert Panel (2007) American Society of Clinical Oncology clinical evidence review on the ongoing care of adult cancer survivors: cardiac and pulmonary late effects. J Clin Oncol 25:3991-4008

El Saiedi SA, Mira MF, Sharaf SA, Al Musaddar MM, El Kaffas RMH, AbdelMassih AF, and Barsoum IHY (2018) Left ventricular diastolic dysfunction without left ventricular hypertrophy in obese children and adolescents: a tissue Doppler imaging and cardiac troponin I study. Cardiol Young 28:76-84.

Ewer MS (2013) Anthracycline cardiotoxicity: clinical aspects, recognition, monitoring, treatment, and prevention, in Cancer and the Heart (Ewer MS and Yeh ET eds) pp 11-41, People's Medical Publishing House, Shelton, CT.
Ewer MS and Lenihan DJ (2008) Left ventricular ejection fraction and cardiotoxicity: is our ear really to the ground? J Clin Oncol 26:1201-1203.

Finkelman BS, Putt M, Wang T, Wang L, Narayan H, Domchek S, DeMichele A, Fox K, Matro J, Shah P, et al. (2017) Arginine-nitric oxide metabolites and cardiac dysfunction in patients with breast cancer. $J$ Am Coll Cardiol 70:152-162.

Fridén V, Starnberg K, Muslimovic A, Ricksten SE, Bjurman C, Forsgard N, Wickman A, and Hammarsten O (2017) Clearance of cardiac troponin $\mathrm{T}$ with and without kidney function. Clin Biochem 50:468-474.

Hillock RJ, Frampton CM, Yandle TG, Troughton RW, Lainchbury JG, and Richards AM (2008) B-type natriuretic peptide infusions in acute myocardial infarction. Heart 94:617-622.

Kilickap S, Barista I, Akgul E, Aytemir K, Aksoyek S, Aksoy S, Celik I, Kes S, and Tekuzman G (2005) cTnT can be a useful marker for early detection of anthracycline cardiotoxicity. Ann Oncol 16:798-804

Lovelock JD, Monasky MM, Jeong EM, Lardin HA, Liu H, Patel BG, Taglieri DM, Gu L, Kumar P, Pokhrel N, et al. (2012) Ranolazine improves cardiac diastolic dysfunction through modulation of myofilament calcium sensitivity. Circ Res 110:841-850.

Masson S, Latini R, Anand IS, Vago T, Angelici L, Barlera S, Missov ED, Clerico A Tognoni G, and Cohn JN; Val-HeFT Investigators (2006) Direct comparison of B-type natriuretic peptide (BNP) and amino-terminal proBNP in a large population of patients with chronic and symptomatic heart failure: the Valsartan Heart Failure (Val-HeFT) data. Clin Chem 52:1528-1538

Menna P, Calabrese V, Armento G, Annibali O, Greco C, Salvatorelli E, Marchesi F, Reggiardo G, and Minotti G (2018) Pharmacology of cardio-oncology: chronotropic and lusitropic effects of B-type natriuretic peptide in cancer patients with early diastolic dysfunction induced by anthracycline or nonanthracycline chemotherapy. J Pharmacol Exp Ther 366:158-168.

Menna P, Salvatorelli E, and Minotti G (2008) Cardiotoxicity of antitumor drugs. Chem Res Toxicol 21:978-989.

Minotti G (2013) Pharmacology at work for cardio-oncology: ranolazine to treat early cardiotoxicity induced by antitumor drugs. J Pharmacol Exp Ther 346:343-349.

Minotti G, Menna P, Salvatorelli E, Cairo G, and Gianni L (2004) Anthracyclines: molecular advances and pharmacologic developments in antitumor activity and cardiotoxicity. Pharmacol Rev 56:185-229.

Minotti G, Salvatorelli E, and Menna P (2010) Pharmacological foundations of cardiooncology. J Pharmacol Exp Ther 334:2-8.

Moltzau LR, Aronsen JM, Meier S, Skogestad J, Ørstavik Ø, Lothe GB, Sjaastad I, Skomedal T, Osnes JB, Levy FO, et al. (2014) Different compartmentation of responses to brain natriuretic peptide and C-type natriuretic peptide in failing rat ventricle. J Pharmacol Exp Ther 350:681-690.

Nagueh SF, Appleton CP, Gillebert TC, Marino PN, Oh JK, Smiseth OA, Waggoner AD, Flachskampf FA, Pellikka PA, and Evangelista A (2009) Recommendations for the evaluation of left ventricular diastolic function by echocardiography. J Am Soc Echocardiogr 22:107-133.

Nagueh SF, Smiseth OA, Appleton CP, Byrd BF III, Dokainish H, Edvardsen T, Flachskampf FA, Gillebert TC, Klein AL, Lancellotti P, et al. Houston, Texas; Oslo, Norway; Phoenix, Arizona; Nashville, Tennessee; Hamilton, Ontario, Canada; Uppsala, Sweden; Ghent and Liège, Belgium; Cleveland, Ohio; Novara, Italy; Rochester, Minnesota; Bucharest, Romania; and St. Louis, Missouri (2016) Recommendations for the evaluation of left ventricular diastolic function by echocardiography: an update from the American Society of Echocardiography and the European Association of Cardiovascular Imaging. Eur Heart J Cardiovasc Imaging 17:1321-1360

Olson RD, Mushlin PS, Brenner DE, Fleischer S, Cusack BJ, Chang BK, and Boucek RJ Jr (1988) Doxorubicin cardiotoxicity may be caused by its metabolite, doxorubicinol. Proc Natl Acad Sci USA 85:3585-3589.

Omland T, Sabatine MS, Jablonski KA, Rice MM, Hsia J, Wergeland R, Landaas S, Rouleau JL, Domanski MJ, Hall C, et al.; PEACE Investigators (2007) Prognostic value of B-type natriuretic peptides in patients with stable coronary artery disease: the PEACE Trial. J Am Coll Cardiol 50:205-214.

Redfield MM (2016) Heart failure with preserved ejection fraction. N Engl J Med 375 1868-1877.

Salvatorelli E, Menna P, Chello M, Covino E, and Minotti G (2018) Low-dose anthracycline and risk of heart failure in a pharmacokinetic model of human myocardium exposure: analog specificity and role of secondary alcohol metabolites. $J$ Pharmacol Exp Ther 364:323-331.

Salvatorelli E, Menna P, Gianni L, and Minotti G (2007) Defective taxane stimulation of epirubicinol formation in the human heart: insight into the cardiac tolerability of epirubicin-taxane chemotherapies. J Pharmacol Exp Ther 320:790-800.

Sandri MT, Cardinale D, Zorzino L, Passerini R, Lentati P, Martinoni A, Martinelli G, and Cipolla CM (2003) Minor increases in plasma troponin I predict decreased left ventricular ejection fraction after high-dose chemotherapy. Clin Chem 49: $248-252$

Serrano JM, González I, Del Castillo S, Muñiz J, Morales LJ, Moreno F, Jiménez R, Cristóbal C, Graupner C, Talavera P, et al. (2015) Diastolic dysfunction following anthracycline-based chemotherapy in breast cancer patients: incidence and predictors. Oncologist 20:864-872.

Springer J, Azer J, Hua R, Robbins C, Adamczyk A, McBoyle S, Bissell MB, and Rose RA (2012) The natriuretic peptides BNP and CNP increase heart rate and electrical conduction by stimulating ionic currents in the sinoatrial node and atria myocardium following activation of guanylyl cyclase-linked natriuretic peptide receptors. J Mol Cell Cardiol 52:1122-1134.

Stone PH (2008) Ranolazine: new paradigm for management of myocardial ischemia myocardial dysfunction, and arrhythmias. Cardiol Clin 26:603-614.

Suter TM and Ewer MS (2013) Cancer drugs and the heart: importance and management. Eur Heart $J$ 34:1102-1111.

Swain SM, Whaley FS, and Ewer MS (2003) Congestive heart failure in patients treated with doxorubicin: a retrospective analysis of three trials. Cancer 97: 2869-2879. 
van Heerebeek L, Hamdani N, Falcão-Pires I, Leite-Moreira AF, Begieneman MP, Bronzwaer JG, van der Velden J, Stienen GJ, Laarman GJ, Somsen A, et al. (2012) Low myocardial protein kinase G activity in heart failure with preserved ejection fraction. Circulation 126:830-839.

Vejpongsa P and Yeh ET (2014) Prevention of anthracycline-induced cardiotoxicity: challenges and opportunities. J Am Coll Cardiol 64:938-945.

Weber M and Hamm C (2006) Role of B-type natriuretic peptide (BNP) and NT-proBNP in clinical routine. Heart 92:843-849.

Zamorano JL, Lancellotti P, Rodriguez Muñoz D, Aboyans V, Asteggiano R, Galderisi M, Habib G, Lenihan DJ, Lip GYH, Lyon AR, et al.; ESC Scientific Document Group (2016) 2016 ESC position paper on cancer treatments and cardiovascular toxicity developed under the auspices of the ESC Committee for Practice Guidelines: the task force for cancer treatments and cardiovascular toxicity of the European Society of Cardiology (ESC). Eur Heart J 37: 2768-2801.

Address correspondence to: Dr. Giorgio Minotti, Department of Medicine, Center for Integrated Research, and Unit of Drug Sciences, University Campus Bio-Medico, Via Alvaro del Portillo 21, 00128 Rome, Italy. E-mail: g.minotti@ unicampus.it 